\title{
Edge States of a Three-dimensional Topological Insulator
}

\author{
Oindrila Deb ${ }^{1}$, Abhiram Soori ${ }^{2}$, and Diptiman Sen $^{1}$ \\ ${ }^{1}$ Centre for High Energy Physics, Indian Institute of Science, Bangalore 560 012, India \\ ${ }^{2}$ Max Planck Institute for the Physics of Complex Systems, Nöthnitzer Str. 38, 01187 Dresden, Germany
}

\begin{abstract}
We use the bulk Hamiltonian for a three-dimensional topological insulator such as $\mathrm{Bi}_{2} \mathrm{Se}_{3}$ to study the states which appear on its various surfaces and along the edge between two surfaces. We use both analytical methods based on the surface Hamiltonians (which are derived from the bulk Hamiltonian) and numerical methods based on a lattice discretization of the bulk Hamiltonian. We find that the application of a potential barrier along an edge can give rise to states localized at that edge. These states have an unusual energy-momentum dispersion which can be controlled by applying a potential along the edge; in particular, the velocity of these states can be tuned to zero. The scattering and conductance across the edge is studied as a function of the edge potential. We show that a magnetic field in a particular direction can also give rise to zero energy states on certain edges. We point out possible experimental ways of looking for the various edge states.
\end{abstract}

PACS numbers: 73.20.-r, 73.40.-c

\section{INTRODUCTION}

The last few years have witnessed extensive studies, both theoretical ${ }^{1-5}$ and experimental ${ }^{6-9}$ of a class of materials called topological insulators (TI). These are materials which, ideally, have only gapped states in the bulk and gapless states on the boundaries which are protected by time-reversal symmetry ${ }^{10,11}$. A twodimensional TI hosts one-dimensional gapless edge states while a three-dimensional TI hosts gapless states on its two-dimensional surfaces. Materials such as $\mathrm{Bi}_{2} \mathrm{Te}_{3}$ and $\mathrm{Bi}_{2} \mathrm{Se}_{3}$ are known to have surfaces which host a single Dirac cone near the $\Gamma$ point of the surface Brillouin zone $^{7-9}$. Many interesting features of the surface states have been studied ${ }^{9-21}$. Some of these studies examined interfaces involving a TI and proximate magnetic or superconducting materials ${ }^{15-23}$. Junctions of different surfaces of TIs (which are sometimes separated by a geometrical step or a magnetic domain wall ${ }^{24-32}$, polyhedral surfaces $^{33}$, and junctions of surfaces of a TI with normal metals or magnetic materials ${ }^{34}$ or superconductors ${ }^{35,36}$ have also been studied. Effects of finite $\operatorname{sizes}^{37-43}$ and different orientations ${ }^{31,32,44-48}$ on the surface states have been studied. Zero energy states at the edges of a thin strip have been studied in Ref. 49 and it has been shown that these remain robust in the presence of Zeeman coupling to a magnetic field. Zero energy surface states produced by a Zeeman field have been studied in Ref. 50 . Finally, transport around different surfaces of a TI in the presence of a magnetic field and the Aharonov-Bohm effect has been experimentally studied in Ref. 51 .

The main motivation of our work is to start from the bulk Hamiltonian of a three-dimensional TI like $\mathrm{Bi}_{2} \mathrm{Se}_{3}$ and study the states which appear at the edge between two cleaved surfaces of the system and the effects of a magnetic field and a barrier potential on such edge states. Although the edge states can be studied more easily starting from the surface Hamiltonians and using some boundary conditions (as we will discuss below), the advantages of obtaining the edge states from the bulk
Hamiltonian are the following.

(i) It would confirm that the edge states are not an artifact of the boundary conditions on the surface Hamiltonians.

(ii) It gives us a better understanding of the physical conditions necessary for the appearance of edge states.

Since analytical calculations starting from the bulk Hamiltonian are not feasible for this problem, we will introduce a lattice discretization of the Hamiltonian and carry out calculations numerically.

The plan of the paper is as follows. In Sec. II we review how the states on the different surfaces of the system can be obtained from a continuum version of the bulk Hamiltonian which involves four bands (including spin) ${ }^{32}$. We will be interested in a system which is infinitely long in, say, the $y$ direction (so that the momentum $k_{y}$ is a good quantum number) and has a cross-section which is a square lying in the $x-z$ plane $^{51}$. Hence the system has four surfaces which are separated pairwise by four infinitely long edges. We show that although the Hamiltonians governing the states on different surfaces have quite different forms (as a result of which the dispersion and spin-momentum locking look quite different), the wave functions on different surfaces can be related by a unitary transformation which conserves the current perpendicular to the edges. We then consider the scattering of the surface states from the edges. In the absence of a potential applied along an edge, we discover that there is no reflection at that edge, but if a potential is applied, there is reflection. We calculate the conductance across an edge as a function of the potential at the edge. Next, we show that states localized along an edge can appear in two situations. If a potential is applied along an edge, edge states appear on that edge. Their wave functions exponentially decay away from that edge into the adjoining surfaces. Rather unusually, the dispersion of these edge states is found to be of the form $E=v\left|k_{y}\right|$, where $k_{y}$ is the momentum along the edge; the spin structure of these states is also different from that of the surface states. Both the sign and magnitude of $v$ can be con- 


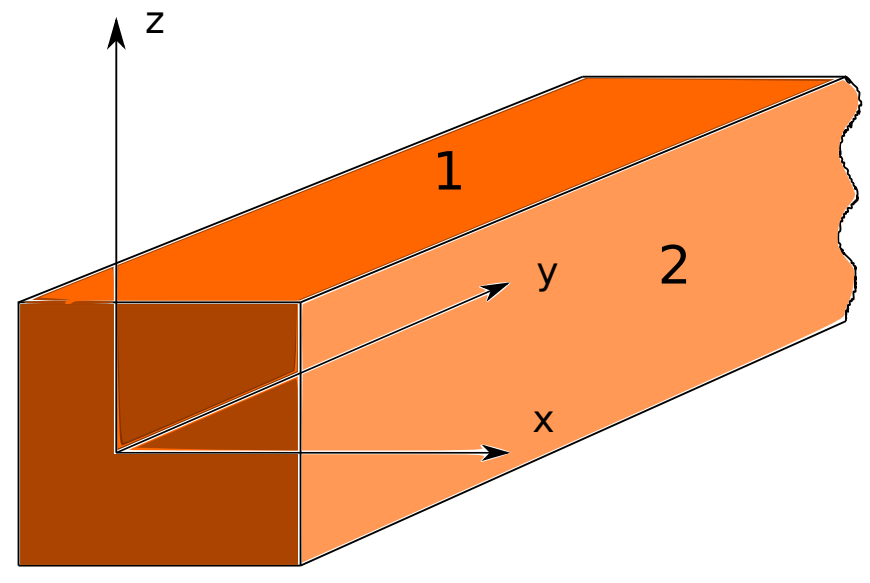

FIG. 1: (Color online) Schematic picture of a system with four surfaces with finite widths and infinite length. The top surface (1), the side surface (2), and the junction between them will be studied in this paper.

trolled by varying the strength of the potential at the edge. In particular, $v$ can be tuned to zero thereby giving rise to edge states with zero group velocity. We also discover that the presence of a magnetic field in the $x$ direction can produce states on two out of the four edges for certain ranges of values of $k_{y}$. These states have zero energy and their spin points along the $z$ direction.

To understand the various edge states better, we then study them numerically as follows. In Sec. III we introduce a lattice discretization of the bulk Hamiltonian in the $x-z$ plane, maintaining the continuum approximation in the $y$ direction. In Sec. IV we present our numerical results for the various states of the system. These are of two types: bulk states which are gapped and surface states which are gapless (in the absence of a magnetic field) with a Dirac spectrum. If a potential is applied along an edge or a magnetic field is applied in the $x$ direction, we discover that a third type of states appear; these are the edge states whose wave functions are localized along an edge and exponentially decay away into both the adjoining surfaces and the bulk. The dispersion and spin structure of these edge states agree qualitatively with that found in Sec. II. In Sec. V, we summarize our work and discuss some possible experiments to test our results.

\section{CONTINUUM MODEL}

In this section, we review how the surface states of a TI like $\mathrm{Bi}_{2} \mathrm{Se}_{3}$ arise from a four-component continuum description of the bulk ${ }^{32}$. We consider a Hamiltonian in the bulk of the form

$$
H_{b}=-m \tau^{z}-i v_{z} \tau^{y} \partial_{z}+i v_{\|} \tau^{x}\left(\sigma^{y} \partial_{x}-\sigma^{x} \partial_{y}\right),
$$

where the constants $m, v_{z}$ and $v_{\|}$are all positive. Here $\sigma^{a}$ denote the Pauli spin matrices, while $\tau^{a}$ denotes pseudospin, with $\tau^{z}= \pm 1$ denoting $B i$ and $S e$ respectively.
(We will set $\hbar=1$ unless mentioned otherwise). In a basis in which $\sigma^{y}$ and $\tau^{y}$ are imaginary and the other four matrices are real, the above Hamiltonian is time-reversal symmetric where the time-reversal transformation complex conjugates all numbers and transforms wave functions as $\psi \rightarrow \sigma^{y} \psi^{*}$.

For a translationally invariant system in which momentum is a good quantum number, the dispersion following from Eq. (1) is given by

$$
E_{k_{x}, k_{y}, k_{z}}= \pm \sqrt{v_{\|}^{2}\left(k_{x}^{2}+k_{y}^{2}\right)+v_{z}^{2} k_{z}^{2}+m^{2}}
$$

which has a gap of $2 m$ at zero momentum.

\section{A. Surface states}

We now examine how surface states arise from Eq. (1). Ref. 32 has discussed this for plane surfaces with arbitrary orientations. A schematic picture of a system with a square cross-section is shown in Fig. 1; the surfaces are infinitely long in the $y$ direction and have a finite width in the other two directions. We will only consider two of the surfaces in this section, the top surface and a side surface, which we label as 1 and 2 respectively, as shown in Fig. 1.

We take the top surface to lie at $z=0$ such that the system fills up the region with $z<0$ and there is vacuum in the region with $z>0$. To define the problem completely, we have to specify the boundary conditions at $z=0$; some possibilities are discussed in Refs. 37-39 and 41. However we will follow Ref. 32; here the vacuum region is also taken to be governed by Eq. (1), except that the parameter $m$ is large and negative there. We write the Hamiltonian as $H_{b}=H_{0}+H^{\prime}$, where

$$
\begin{aligned}
H_{0} & =-m(z) \tau^{z}-i v_{z} \tau^{y} \partial_{z}, \\
H^{\prime} & =i v_{\|} \tau^{x}\left(\sigma^{y} \partial_{x}-\sigma^{x} \partial_{y}\right),
\end{aligned}
$$

where $m(z)$ is a positive (negative) constant for $z<0$ $(z>0)$ as stated above. Next, we note that $H_{0}$ has a zero energy eigenvector which is localized near the surface; the corresponding wave function is given by

$$
\Psi_{1}(x, y, z, t)=\psi_{1}(x, y, t) \exp \left[\frac{1}{v_{z}} \int_{0}^{z} d z^{\prime} m\left(z^{\prime}\right)\right],
$$

where $H_{0} \Psi_{1}=0$ implies that the four-component spinor $\psi_{1}$ must satisfy

$$
\left(\tau^{z}+i \tau^{y}\right) \psi_{1}=0 .
$$

The identity $\left(\tau^{z}-i \tau^{y}\right)\left(\tau^{z}+i \tau^{y}\right)=2\left(I+\tau^{x}\right)$ implies that all solutions of Eq. (5) must satisfy the condition $\tau^{x} \psi_{1}=-\psi_{1}$. We now observe that $H^{\prime}$ commutes with $\tau^{x}$. The condition $\tau^{x} \psi_{1}=-\psi_{1}$ then implies that the equation $H^{\prime} \psi_{1}=E \psi_{1}$ can be written as $H_{s 1} \psi_{1}=E \psi_{1}$, where

$$
H_{s 1}=-i v_{\|}\left(\sigma^{y} \partial_{x}-\sigma^{x} \partial_{y}\right)
$$


is the Hamiltonian governing the states at the top surface. We now see that the solutions of $H_{b} \Psi_{1}=E \Psi_{1}$ which are localized near the top surface must be of the form given in Eq. (4), where $\psi_{1}=u_{1} \exp \left[i\left(k_{x} x+k_{y} y-\right.\right.$ $E t)]$

$$
E= \pm \sqrt{v_{\|}^{2}\left(k_{x}^{2}+k_{y}^{2}\right)}
$$

and $u_{1}$ satisfies

$$
\begin{aligned}
v_{\|}\left(\sigma^{y} k_{x}-\sigma^{x} k_{y}\right) u_{1} & =E u_{1}, \\
\tau^{x} u_{1} & =-u_{1} .
\end{aligned}
$$

In the basis in which $\sigma^{z}$ and $\tau^{z}$ are diagonal matrices with the diagonal elements being given by $\sigma^{z}=(1,-1,1,-1)$ and $\tau^{z}=(1,1,-1,-1)$, we can show that the normalized solution of the above equations with $E>0$ takes the form $u_{1}=(1 / 2)\left(1,\left(i k_{x}-k_{y}\right) / k,-1,\left(-i k_{x}+k_{y}\right) / k\right)^{T}$, where $k=\sqrt{k_{x}^{2}+k_{y}^{2}}$. We then find that the expectation value of the spin, $\vec{S}=\vec{\sigma} / 2$, in this state is given by

$$
\frac{1}{2} \psi_{1}^{\dagger} \vec{\sigma} \psi_{1}=\frac{1}{2} \hat{z} \times \hat{k}
$$

This relation between $\langle\vec{S}\rangle$ and $\hat{k}$ is called spin-momentum locking.

Next, we can derive an expression for the current $\vec{J}$ by using the Dirac equation $i \partial \psi_{1} / \partial t=H_{s 1} \psi_{1}$ and the equation of continuity, $\partial \rho / \partial t+\vec{\nabla} \cdot \vec{J}=0$, where the charge density is given by $\rho=\psi_{1}^{\dagger} \psi_{1}$. We then find that the state with $E>0$ has a current along the $x$ direction given by

$$
\begin{aligned}
\hat{x} \cdot \vec{J} & =v_{\|} \psi_{1}^{\dagger} \sigma^{y} \psi_{1} \\
& =v_{\|} \frac{v_{\|} k_{x}}{E}
\end{aligned}
$$

Similarly we can consider a side surface lying at $x=0$ such that the system (vacuum) lies in the region with $x<$ $0(x>0)$. The Hamiltonian in Eq. (1) is taken to be valid in both regions with $m$ being a positive (large negative) constant respectively. We write the Hamiltonian as $H_{b}=$ $H_{0}+H^{\prime}$, where

$$
\begin{aligned}
H_{0} & =-m(z) \tau^{z}+i v_{\|} \sigma^{y} \tau^{x} \partial_{x} \\
H^{\prime} & =-i\left(v_{z} \tau^{y} \partial_{z}+v_{\|} \sigma^{x} \tau^{x} \partial_{y}\right) .
\end{aligned}
$$

Now $H_{0}$ has a zero energy eigenvector localized near the surface, with a wave function given by

$$
\Psi_{2}(x, y, z, t)=\psi_{2}(y, z, t) \exp \left[\frac{1}{v_{\|}} \int_{0}^{x} d x^{\prime} m\left(x^{\prime}\right)\right],
$$

where $\psi_{2}$ is a four-component spinor which satisfies

$$
\left(\tau^{z}-i \sigma^{y} \tau^{x}\right) \psi_{2}=0
$$

The identity $\left(\tau^{z}+i \sigma^{y} \tau^{x}\right)\left(\tau^{z}-i \sigma^{y} \tau^{x}\right)=2\left(I+\sigma^{y} \tau^{y}\right)$ implies that all solutions of Eq. (15) must satisfy the condition $\sigma^{y} \tau^{y} \psi_{2}=-\psi_{2}$. Combining this condition with the fact that $H^{\prime}$ commutes with $\sigma^{y} \tau^{y}$ shows that the equation $H^{\prime} \psi_{2}=E \psi_{2}$ can be written as $H_{s 2} \psi_{2}=E \psi_{2}$, where

$$
H_{s 2}=i\left(v_{z} \sigma^{y} \partial_{z}-v_{\|} \sigma^{z} \tau^{z} \partial_{y}\right),
$$

is the Hamiltonian on the side surface. (In deriving Eq. (16), we have used the relations $\sigma^{y}\left(\sigma^{y} \tau^{y}\right)=\tau^{y}$ and $\left.\sigma^{z} \tau^{z}\left(\sigma^{y} \tau^{y}\right)=-\sigma^{x} \tau^{x}\right)$. Thus, the solutions of $H_{b} \Psi_{2}=E \Psi_{2}$ which are localized near the side surface must be of the form given in Eq. (14), where $\psi_{2}=$ $u_{2} \exp \left[i\left(k_{y} y+k_{z} z-E t\right)\right]$,

$$
E= \pm \sqrt{v_{z}^{2} k_{z}^{2}+v_{\|}^{2} k_{y}^{2}}
$$

and

$$
\begin{aligned}
\left(-v_{z} \sigma^{y} k_{z}+v_{\|} \sigma^{z} \tau^{z} k_{y}\right) u_{2} & =E u_{2}, \\
\sigma^{y} \tau^{y} u_{2} & =-u_{2} .
\end{aligned}
$$

We find that the normalized solution of these equations with $E>0$ has an expectation value of the spin given by

$$
\frac{1}{2} \psi_{2}^{\dagger} \vec{\sigma} \psi_{2}=-\frac{v_{z} k_{z}}{2 E} \hat{y}
$$

and a current along the $z$ direction given by

$$
\begin{aligned}
\hat{z} \cdot \vec{J} & =-v_{z} \psi_{2}^{\dagger} \sigma^{y} \psi_{2} \\
& =v_{z} \frac{v_{z} k_{z}}{E}
\end{aligned}
$$

\section{B. Edge between two surfaces}

We now consider a system which has semi-infinite surfaces given by $(z=0, x<0)$ and $(x=0, z<0)$; these meet along the infinitely long edge $x=z=0$ which runs along the $y$ direction. The system lies in the region $x<0$ and $z<0$. In this subsection we will ignore the bulk Hamiltonian and work with only the surface Hamiltonians given in Eqs. (6) and (16). This implicitly assumes that the energy scales of the surface states, such as $v_{\|}|\vec{k}|$ and $v_{z}|\vec{k}|$, are both much smaller than the bulk gap. We will study two problems, one involving scattering of electrons incident on the edge from one of the surfaces, and the other involving the existence of states localized along the edges (discussed in the next section). For this purpose we first need to find the matching condition at the edge between the wave functions on the top and side surfaces.

We saw above that the surface Hamiltonians and conditions have two different forms, Eqs. (6),(9) and (16),(19), on the two surfaces. The current perpendicular to the edge and coming into it on the top surface is given by

$$
\hat{x} \cdot \vec{J}=v_{\|} \psi_{1}^{\dagger} \sigma^{y} \psi_{1}
$$


while the current going away from the edge on the side surface is given by

$$
-\hat{z} \cdot \vec{J}=v_{z} \psi_{2}^{\dagger} \sigma^{y} \psi_{2} .
$$

For a plane wave incident on the edge from either surface, the conservation of current perpendicular to the edge implies that $\hat{x} \cdot \overrightarrow{J_{1}}=-\hat{z} \cdot \vec{J}$, namely,

$$
v_{\|}\left(\psi_{1}^{\dagger} \sigma^{y} \psi_{1}\right)_{x=0}=v_{z}\left(\psi_{2}^{\dagger} \sigma^{y} \psi_{2}\right)_{z=0} .
$$

This implies that the wave functions are related as

$$
\left(\psi_{2}\right)_{z=0}=\sqrt{\frac{v_{\|}}{v_{z}}} U\left(\psi_{1}\right)_{x=0},
$$

where the matrix $U$ must satisfy

$$
U^{\dagger} \sigma^{y} U=\sigma^{y} .
$$

In addition, we require that

$$
U^{-1} \sigma^{y} \tau^{y} U=\tau^{x}
$$

for the consistency of Eqs. (9) and (19), and

$$
\sigma^{y} U^{*} \sigma^{y}=U
$$

in order to maintain time-reversal symmetry.

We find that the most general solution of Eqs. (27-29) involves two real parameters and is given by

$$
U=e^{-i\left(\beta \sigma^{y}+\gamma \tau^{y}\right)} e^{i(3 \pi / 4) \sigma^{y} \tau^{z}} .
$$

(This implies that $U$ is a real and unitary matrix). However, we can bring the factor of $e^{-i\left(\beta \sigma^{y}+\gamma \tau^{y}\right)}$ to the left hand side of Eq. (26) and use the condition that $\sigma^{y} \tau^{y} \psi_{2}=-\psi_{2}$ to show that $e^{-i\left(\beta \sigma^{y}+\gamma \tau^{y}\right)} \psi_{2}=$ $e^{-i(\beta-\gamma) \sigma^{y}} \psi_{2}$. The general boundary condition satisfying Eqs. (26-29) therefore only has one real parameter $\alpha=\beta-\gamma$ and is given by

$$
\left(\psi_{2}\right)_{z=0}=\sqrt{\frac{v_{\|}}{v_{z}}} e^{-i \alpha \sigma^{y}} e^{i(3 \pi / 4) \sigma^{y} \tau^{z}}\left(\psi_{1}\right)_{x=0} .
$$

We note that related matching conditions, but without the parameter $\alpha$, have been discussed in Ref. 48 and in a TI system with a different geometry in Ref. 40. We will see below that the parameter $\alpha$ leads to some non-trivial effects, namely, scattering at the edge and the appearance of states localized at the edge; such effects therefore do not appear in Ref. 48.

The parameter $\alpha$ in Eq. (31) can be given a precise physical interpretation as was shown in Ref. 25. Consider a $\delta$-function potential barrier placed along the edge at $x=-\epsilon$, where $\epsilon$ is an infinitesimal positive quantity. If the $\delta$-function potential is given by $V_{0} \delta(x+\epsilon)$, one can use the Hamiltonian in Eq. (6) to integrate through this potential to show that the wave function has a discontinuity given by ${ }^{25}$

$$
\left(\psi_{1}\right)_{x=0}=e^{-i \alpha \sigma^{y}}\left(\psi_{1}\right)_{x=-2 \epsilon},
$$

where $\alpha=V_{0} / v_{\|}$. This gives us an understanding of the parameter $\alpha$. If there is no potential barrier along the edge, we must set $\alpha=0$ in Eq. (31). Note that the presence of a $\delta$-function potential barrier produces a discontinuity in a wave function satisfying the Dirac equation, in contrast to the Schrödinger equation where the wave function remains continuous but its first derivative becomes discontinuous.

We now consider the problem of scattering from the edge. Since there is translational invariance along the edge, the momentum $k_{y}$ along that direction will be conserved. We assume that an electron with energymomentum $\left(E, k_{x}, k_{y}\right)$, satisfying Eq. (7), is incident on the edge from the top surface; for definiteness, we assume that $E>0$ and $k_{x}>0$. The angle of incidence, $\theta_{i}=\tan ^{-1}\left(k_{y} / k_{x}\right)$, can vary from $-\pi / 2$ to $\pi / 2$. The electron will then be reflected in the top surface with amplitude $r$ and energy-momentum $\left(E,-k_{x}, k_{y}\right)$, and transmitted to the side surface with amplitude $t$ and energymomentum $\left(E, k_{z}, k_{y}\right)$ satisfying Eq. (17). The conservation of $E$ and $k_{y}$ imply that $v_{z} k_{z}=-v_{\|} k_{x}$; the minus sign arises because we require $k_{z}<0$ so that the transmitted electron moves away from the edge. If $\psi_{i}, \psi_{r}$ and $\psi_{t}$ denote the normalized four-component spinors corresponding to the incident, reflected and transmitted waves respectively, then they will satisfy the boundary condition (Eq. (31))-

$$
t \psi_{t}=\sqrt{\frac{v_{\|}}{v_{z}}} e^{-i \alpha \sigma^{y}} e^{i(3 \pi / 4) \sigma^{y} \tau^{z}}\left(\psi_{i}+r \psi_{r}\right)
$$

at the edge. In general $r$ and $t$ will be functions of $\vec{k}, E$ and $\alpha$. It is clear from Eq. (33) that changing $\alpha \rightarrow \alpha+\pi$ only changes the sign of $t$, leaving $|t|^{2}$ unchanged. Hence $|t|^{2}$ is a periodic function of $\alpha$ with period $\pi$, and it is sufficient to consider $\alpha$ to lie in the range $[-\pi / 2, \pi / 2]$. Eq. (25) implies that the reflected and transmitted currents will satisfy current conservation,

$$
v_{\|}\left(1-|r|^{2}\right)=v_{z}|t|^{2} .
$$

In momentum space, the Hamiltonian governing the incident wave on the top surface is given by

$$
H_{s 1}=v_{\|}\left(\sigma^{y} k_{x}-\sigma^{x} k_{y}\right),
$$

while the Hamiltonian governing the transmitted wave on the side surface is given by

$$
H_{s 2}=v_{\|}\left(\sigma^{y} k_{x}+\sigma^{z} \tau^{z} k_{y}\right),
$$

where we have used the relation $v_{z} k_{z}=-v_{\|} k_{x}$. We now note that

$$
H_{s 2}=e^{i(3 \pi / 4) \sigma^{y} \tau^{z}} H_{s 1} e^{-i(3 \pi / 4) \sigma^{y} \tau^{z}} .
$$

This implies that if $\alpha=0$, then $r=0$ and $t=\sqrt{v_{\|} / v_{z}}$ will satisfy both the Dirac equations, $H_{s 1} \psi_{i}=E \psi_{i}$ and $H_{s 2} \psi_{t}=E \psi_{t}$, and the boundary condition in Eq. (33). This will be true regardless of the angle of incidence. A 
similar argument holds if the electron is incident from the side surface. We therefore conclude that if there is no barrier potential at the edge, there will be no reflection and a wave incident from either surface will transmit perfectly to the other surface.

We would like to mention here that our result that there is no reflection in the absence of a barrier potential differs from the one presented in Ref. 25. The reason for this difference is that the analysis in Ref. 25 simply assumed certain two-component Dirac equations on the two surfaces without considering where these equations come from, whereas our present analysis begins from a fourcomponent bulk Hamiltonian which appears in a specific system such as $\mathrm{Bi}_{2} \mathrm{Se}_{3}$. Thus our analysis shows that the bulk Hamiltonian gives rise to some Dirac equations on the two surfaces which are compatible with each other in such a way that there is no reflection.

The absence of reflection when there is no barrier potential has an implication for the conductance across the edge. The conductance can be derived as follows. Assuming that the system has a large width in the $y$ direction given by $W$, the current going from the top surface to the side surface is given by

$$
I=e W \iint \frac{d k_{x} d k_{y}}{(2 \pi)^{2}}(-\hat{z} \cdot \vec{J})|t|^{2},
$$

where $e$ is the charge of an electron. Eq. (22) implies that $-\hat{z} \cdot \vec{J}=-v_{z}\left(v_{z} k_{z} / E\right)=v_{z}\left(v_{\|} k_{x} / E\right)=v_{z} \cos \theta_{i}($ we recall that $\left.k_{z}<0\right)$, where $\theta_{i}$ is the angle of incidence and $E=\sqrt{v_{\|}^{2}\left(k_{x}^{2}+k_{y}^{2}\right)}=\sqrt{v_{z}^{2} k_{z}^{2}+v_{\|}^{2} k_{y}^{2}}$. We now rewrite $d k_{x} d k_{y}=\left(E / v_{\|}^{2}\right) d E d \theta_{i}$. The integral over $E$ goes from $\mu_{1}$ to $\mu_{2}$ which are the Fermi energies (with respect to the Dirac point) in the electron reservoirs connected to the top and side surfaces. The voltage applied to a reservoir $i$ is related to its Fermi energy as $\mu_{i}=e V_{i}$. The differential conductance $G=\frac{d I}{d V}$ is the ratio of the change in current $d I$ to the change in bias $d V$ when the Fermi energy of TI-1 is changed from $\mu=e V$ to $\mu+e d V$. This is given by

$$
G=\frac{e^{2} W \mu}{v_{\|}^{2}(2 \pi \hbar)^{2}} \int_{-\pi / 2}^{\pi / 2} d \theta_{i} v_{z} \cos \theta_{i}|t|^{2},
$$

where the combination $\mu /\left(v_{\|}^{2} \hbar^{2}\right)$ on the right hand side comes from the density of states of a massless Dirac electron with Fermi energy $\mu$, and we have restored factors of $\hbar$ here. For the reflectionless case, we have $|t|^{2}=v_{\|} / v_{z}$. We then find that $G=G_{0}$ where

$$
G_{0}=\frac{2 e^{2} W \mu}{v_{\|}(2 \pi \hbar)^{2}} \text {. }
$$

The factor of $1 / v_{\|}$in Eq. (40) can be traced back to the fact that we are calculating the conductance across the edge which runs along the $y$ direction, and the velocity in that direction is $v_{\|}$. In general, if the velocities along the $x, y$ and $z$ directions are $v_{x}, v_{y}$ and $v_{z}$ respectively, the differential conductance would be proportional to $1 / v_{y}$; this would come from a product of the density of states for the incident waves on the top surface, the probability of transmission to the side surface and the transmitted current perpendicular to the edge on the side surface which are proportional to $E /\left(v_{x} v_{y}\right), v_{x} / v_{z}$ and $v_{z}$ respectively.

We now study what happens if there is a barrier potential along the edge, i.e., $\alpha \neq 0$ in Eq. (33). For a wave incident on the top surface with energy $E>0$ and angle $\theta_{i}$, we find that the transmission amplitude is given by

$$
\begin{aligned}
t= & i \sqrt{\frac{v_{\|}}{v_{z}}} \frac{2 \sqrt{2} \cos \theta_{i} \sqrt{1-\sin \theta_{i}}}{A+i e^{-i \theta_{i}} B}, \\
A= & (\sin \alpha+\cos \alpha)\left(-i \cos \theta_{i}\right) \\
& +(\sin \alpha-\cos \alpha)\left(1-\sin \theta_{i}\right), \\
B= & (\sin \alpha-\cos \alpha)\left(-i \cos \theta_{i}\right) \\
& -(\sin \alpha+\cos \alpha)\left(1-\sin \theta_{i}\right) .
\end{aligned}
$$

Interestingly, $t$ only depends on the velocity ratio $v_{\|} / v_{z}$ through the prefactor $\sqrt{v_{\|} / v_{z}}$. We can calculate the differential conductance using Eqs. (39) and (41). In Fig. 2 we show a plot of $G / G_{0}$ as a function $\alpha$ lying in the range $[-\pi / 2, \pi / 2]$. It has a maximum value of 1 at $\alpha=0$ and a minimum value of $2 / 3$ at $\alpha= \pm \pi / 2$.

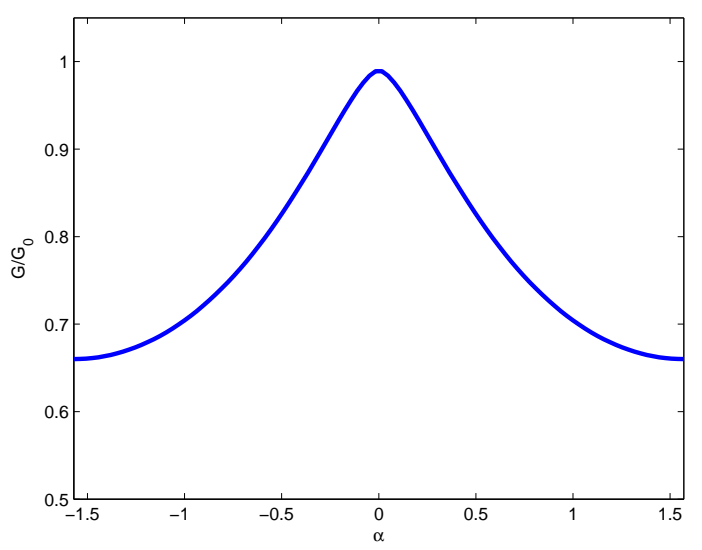

FIG. 2: (Color online) Differential conductance $G / G_{0}$ versus $\alpha$.

\section{Edge states}

In this section, we examine if there are states which are localized along the edge. These have a momentum $k_{y}$ along the $y$ direction, but their wave functions decay as $e^{x / \xi_{1}}$ and $e^{z / \xi_{2}}$ as we go away from edge on the top surface and the side surface respectively. A similar problem has been discussed in Ref. 25 where only the surface Hamiltonians were considered, except that we now have to impose the conditions given in Eqs. (9), (19) and (31). 
We find that the edge states have an unusual dispersion of the form $E=v\left|k_{y}\right|$, where $v$ depends on the barrier parameter $\alpha$. (We observe that this differs from a chiral dispersion where $E / k_{y}$ has the same sign for both positive and negative values of $k_{y}$ ). We find that $v=v_{\|} \cos \alpha>0$ if $-\pi / 2 \leq \alpha<0$ and $v=-v_{\|} \cos \alpha<0$ if $0<\alpha \leq \pi / 2$. There are no edge states if $\alpha=0$; thus the absence of edge states goes hand in hand with the absence of reflection for the scattering problem. If $\alpha= \pm \pi / 2$, the bound states form a flat band with $E=0$. The decay lengths $\xi_{i}$ turn out to be inversely proportional to $\left|k_{y}\right|$; in particular, we find that $\xi_{1}=1 /\left|k_{y} \sin \alpha\right|$ and $\xi_{2}=\left(v_{z} / v_{\|}\right) \xi_{1}$. There is no edge state at $k_{y}=0$ where $\xi_{i} \rightarrow \infty$. Finally, the expectation value of the spin of the edge states lies in the $x-z$ plane and points in a direction which depends on $\alpha$ and the signs (but not the magnitudes) of $k_{y}$ and $E$. For instance, if $k_{y}, E>0$, we find that the spin points in the direction $-\cos \alpha \hat{x}-\sin \alpha \hat{z}$.

Interestingly, we discover that edge states also appear if a uniform magnetic field is applied along the $x$ direction and there is no potential barrier present. A magnetic field adds two terms to the Hamiltonian: (i) a Zeeman coupling $-\left(g \mu_{B} / 2\right) \vec{\sigma} \cdot \vec{B}$, where $g$ is the gyromagnetic ratio (which we will take equal to 2 ) and $\mu_{B}=e \hbar /\left(2 m_{e} c\right)$ is the Bohr magneton, and (ii) an orbital term where we change the momentum operator $-i \vec{\nabla} \rightarrow-i \vec{\nabla}-(e / c) \vec{A}$, where $\vec{A}$ is the vector potential. For a magnetic field in the $x$ direction, the Zeeman term is given by $-\left(g \mu_{B} / 2\right) B_{x} \sigma^{x}$. It is convenient to define

$$
b_{x}=g \mu_{B} B_{x}=\frac{e \hbar}{m_{e} c} B_{x}
$$

which has the dimensions of energy. We will assume that both $v_{\|} k_{y}$ and $b_{x}$ are small compared to the bulk gap, so that we can use only the surface Hamiltonians. Eq. (20) implies that the wave functions on the side surface have the expectation value $\left\langle\sigma^{x}\right\rangle=0$. Hence, to first order in $b_{x}$, we will ignore the term proportional to $b_{x} \sigma^{x}$ on the side surface and keep it only on the top surface. Turning to the orbital term, we can choose the vector potential to be $\vec{A}=-B_{x} z \hat{y}$. On the top surface, the vector potential vanishes since $z=0$; hence we keep the vector potential only on the side surface. We will therefore work with the Hamiltonians

$$
\begin{aligned}
& H_{s 1}=-i v_{\|}\left(\sigma^{y} \partial_{x}-\sigma^{x} \partial_{y}\right)-\frac{1}{2} b_{x} \sigma^{x}, \\
& H_{s 2}=i v_{z} \sigma^{y} \partial_{z}+v_{\|} \sigma^{z} \tau^{z}\left(-i \partial_{y}+\frac{e}{c} B_{x} z\right),
\end{aligned}
$$

on the top and side surfaces respectively. We have to impose the conditions in Eqs. (9), (19) and (31) (with $\alpha=0$ ). Once again we will look for eigenstates with momentum $k_{y}$ along the $y$ direction and energy $E$. We then discover that there are solutions with zero energy with wave functions given by

$$
\begin{aligned}
& \psi_{1}=u_{1} e^{i\left(k_{y} y-E t\right)+x / \xi_{1}}, \\
& \psi_{2}=u_{2} e^{i\left(k_{y} y-E t\right)+z / \xi_{2}+\operatorname{sgn}\left(k_{y}\right)\left(e B_{x} v_{\|} / 2 c v_{z}\right) z^{2}},
\end{aligned}
$$

on the top and side surfaces (here sgn denotes the signum function), provided that

$$
b_{x}=-2(\lambda+1) v_{\|} k_{y},
$$

where $\lambda$ is positive. Namely, the magnitude of $b_{x}$ must be larger than $2 v_{\|}\left|k_{y}\right|$, and its sign must be opposite to $k_{y}$. The decay lengths are given by

$$
\xi_{1}=\frac{2 v_{\|}}{\left|b_{x}+2 v_{\|} k_{y}\right|} \quad \text { and } \quad \xi_{2}=\frac{v_{z}}{v_{\|}\left|k_{y}\right|} .
$$

The forms of the spinors $u_{1}, u_{2}$ in Eqs. (44-45) depend only on the sign of $k_{y}$, and not on the magnitude of $k_{y}$ or $b_{x}$. For instance, for $k_{y}>0$ and $b_{x}<-2 v_{\|}\left|k_{y}\right|$, we find that

$$
u_{1}=\frac{1}{\sqrt{2}}\left(\begin{array}{c}
0 \\
1 \\
0 \\
-1
\end{array}\right) \quad \text { and } \quad u_{2}=\frac{1}{2}\left(\begin{array}{c}
-1 \\
1 \\
-1 \\
-1
\end{array}\right) .
$$

Eqs. (48) show that the $u_{1}^{\dagger} \vec{\sigma} u_{1}=-\hat{z}$ and $u_{2}^{\dagger} \vec{\sigma} u_{2}=0$. Hence this state will have the expectation value of $\vec{S}$ pointing in the $-\hat{z}$ direction. If $k_{y}<0$ and $b_{x}>2 v_{\|}\left|k_{y}\right|$, the edge state will have $\vec{S}$ pointing in the $+\hat{z}$ direction. Note that the edge states that appear at the junction are mainly due to the Zeeman field. We have included the orbital term only for the sake of completeness.

If the magnitude of $b_{x}$ is larger than $2 v_{\|}\left|k_{y}\right|$, but its sign is the same as $k_{y}$, edge states do not appear along the junction of the top and side surface but they appear along the junction of other pairs of surfaces (such as the bottom surface and the other side surface shown in Fig. 1). This can be shown by first deriving the Hamiltonians on the other surfaces and then carrying out an analysis similar to the one give above.

The zero energy states discussed here occur for reasons which are similar to the zero energy state which appears in a one-dimensional Dirac equation if the mass changes sign at one point ${ }^{52}$. For a given value of $k_{y}$, we can think of the two Hamiltonians in Eqs. (43) as being unitarily equivalent to a one-dimensional Dirac equation with a mass term proportional to $v_{\|} k_{y}+b_{x} / 2$ on one side (the top surface) and to $v_{\|} k_{y}$ on the other side (the side surface). (We are ignoring the orbital term $(e / c) B_{x} z$ here). A zero energy state appears precisely when $v_{\|} k_{y}+b_{x} / 2$ and $v_{\|} k_{y}$ have opposite signs as we see from Eq. (46) when $\lambda>0$.

The discussion in this section for the various edge states was based entirely on the surface Hamiltonians and ignored the bulk Hamiltonian. We would now like to study these states more carefully by taking the bulk Hamiltonian into account. In the continuum, this is a difficult problem to study analytically since the edge states will decay into both the surfaces as well as into the bulk. Further, the bulk Hamiltonian contains terms of higher order in the momenta as we will see in Eq. (49) below; this too makes an analytical calculation difficult. We will therefore study this problem numerically. A convenient way of doing this is to consider a lattice version of the model. This will be the subject of Secs. III and IV. 


\section{LATTICE MODEL}

In this section we will present and numerically study a lattice model for the bulk of the system. The lattice model will be used only as a way of discretizing the bulk Hamiltonian; the lattice that we will introduce is not the same as the original microscopic lattice of $\mathrm{Bi}_{2} \mathrm{Se}_{3}$. A lattice model has several advantages ${ }^{55}$ : we can obtain explicit expressions for the wave functions, both near the surface and in the bulk, and we do not have to impose any conditions such as Eqs. (9), (19) and (31).

In momentum space, a continuum Hamiltonian for $\mathrm{Bi}_{2} \mathrm{Se}_{3}$ in the bulk is given by ${ }^{11}$

$$
\begin{aligned}
H_{b}= & -\left[m-B_{1} k_{z}^{2}-B_{2}\left(k_{x}^{2}+k_{y}^{2}\right)\right] \tau^{z} \\
& +v_{z} \tau^{y} k_{z}+v_{\|} \tau^{x}\left(\sigma^{x} k_{y}-\sigma^{y} k_{x}\right),
\end{aligned}
$$

up to second order in $\vec{k}$ close to the $\Gamma$ point of the threedimensional Brillouin zone, where $m=0.28 \mathrm{eV}, B_{1}=$ $6.86 \mathrm{eV} \AA^{2}, B_{2}=44.5 \mathrm{eV} \AA^{2}, v_{z}=2.26 \mathrm{eV} \AA$, and $v_{\|}=$ $3.33 \mathrm{eV \AA}$. Note that if we only keep terms up to first order in $\vec{k}$, Eq. (49) reduces to Eq. (1). Ref. 11 gives some additional terms which are proportional to the identity matrix and which break particle-hole symmetry; we will ignore these terms in the calculations presented here.

We will now introduce a lattice discretization of Eq. (49) as follows. Since we want to study a system with an infinitely long edge along the $y$ direction, we will describe the system using a square lattice in the $x-z$ plane and a continuum model in the $y$ direction where the electrons have momentum $k_{y}$. Each site of the square lattice will have four components corresponding to $\mathrm{Bi}$ and Se with $S^{z}= \pm 1 / 2$; the sites will be labeled by two integers $\left(n_{x}, n_{z}\right)$. The lattice spacing will be taken to be $a=9.94 \AA$ which is the height of a single quintuple layer of $\mathrm{Bi}_{2} \mathrm{Se}_{3}{ }^{5}$. We now define a lattice Hamiltonian which reduces to Eq. (49) in the limit $k_{x} a, k_{z} a \rightarrow 0$. This can be done by replacing $k_{x} \rightarrow(1 / a) \sin \left(k_{x} a\right)$, $k_{x}^{2} \rightarrow\left(2 / a^{2}\right)\left[1-\cos \left(k_{x} a\right)\right]$, and similarly for $k_{z}, k_{z}^{2}$. We emphasize that we are using the lattice only to discretize the bulk Hamiltonian in Eq. (49) which is valid close to zero momentum (the $\Gamma$ point). Namely, we will only study long wavelength modes using our lattice model. Hence our results can only be trusted for those states whose length scales (such as the decay lengths of the edge states) are much larger than the lattice spacing. In this limit, the exact structure of the lattice is not important.

We also want to study the effects of a potential $V_{n_{x}, n_{z}}$ placed on certain sites of the lattice, and a uniform magnetic field. The magnetic field will have both a Zeeman coupling to the electron spin and an orbital part. In our numerical studies, we will only consider the effect of the Zeeman coupling for the following reason. Since the edge states that we will study are localized along one of the edges and are therefore quasi-one-dimensional, they will not be affected significantly by the orbital term. We also note that a magnetic field that has only a Zeeman coupling and no orbital coupling can be realized in a TI by doping with magnetic impurities ${ }^{53}$ or by depositing a ferromagnetic layer on the surface ${ }^{54}$.

Putting everything together, the eigenvalue equation on the lattice for electrons with momentum $k_{y}$ takes the form

$$
\begin{aligned}
& -\left(m-\frac{2 B_{1}}{a^{2}}-\frac{2 B_{2}}{a^{2}}\right) \tau^{z} \psi_{n_{x}, n_{z}, k_{y}} \\
& +B_{2} k_{y}^{2} \tau^{z} \psi_{n_{x}, n_{z}, k_{y}} \\
& -\frac{B_{1}}{a^{2}} \tau^{z}\left(\psi_{n_{x}, n_{z}+1, k_{y}}+\psi_{n_{x}, n_{z}-1, k_{y}}\right) \\
& -\frac{B_{2}}{a^{2}} \tau^{z}\left(\psi_{n_{x}+1, n_{z}, k_{y}}+\psi_{n_{x}-1, n_{z}, k_{y}}\right) \\
& -\frac{i v_{z}}{2 a} \tau^{y}\left(\psi_{n_{x}, n_{z}+1, k_{y}}-\psi_{n_{x}, n_{z}-1, k_{y}}\right) \\
& +\frac{i v_{\|}}{2 a} \tau^{x} \sigma^{y}\left(\psi_{n_{x}+1, n_{z}, k_{y}}-\psi_{n_{x}-1, n_{z}, k_{y}}\right) \\
& +v_{\|} \tau^{x} \sigma^{x} k_{y} \psi_{n_{x}, n_{z}, k_{y}} \\
& +\left(V_{n_{x}, n_{z}}-\frac{g \mu_{B}}{2} \vec{\sigma} \cdot \vec{B}\right) \psi_{n_{x}, n_{z}, k_{y}} \\
& =E \psi_{n_{x}, n_{z}, k_{y}},
\end{aligned}
$$

where $\psi_{n_{x}, n_{z}, k_{y}}$ denotes the four-component wave function for the electron. It is interesting to note that the terms involving $B_{1}$ and $B_{2}$ help to avoid the problem of fermion doubling (i.e., unwanted low-energy modes) at $k_{x}=\pi / a$ and $k_{z}=\pi / a$ in the bulk spectrum.

It is useful to understand the symmetries of this system. For a given momentum $k_{y}$ in the $y$ direction and energy $E$, the eigenstate $\psi$ will be given by $e^{i\left(k_{y} y-E t\right)}$ times $u\left(n_{x}, n_{z} ; k_{y}, E\right)$ where $u$ is a four-component spinor. Assuming that the magnetic field $\vec{B}$ is zero, we find that Eq. (50) has the following symmetries.

(i) Time-reversal symmetry $\mathcal{T}$ : Eq. (50) remains invariant if we complex conjugate all numbers, and transform $k_{y} \rightarrow-k_{y}$ and $u\left(n_{x}, n_{z}, k_{y}\right) \rightarrow \sigma^{y} u^{*}\left(n_{x}, n_{z},-k_{y}\right)$. It follows that each energy eigenvalue $E$ will appear with a double degeneracy corresponding to momenta $+k_{y}$ and $-k_{y}$. Since $\sigma^{y *}=-\sigma^{y}$, we have $\mathcal{T}^{2}=-I$; this implies that there will be a two-fold Kramers degeneracy for $k_{y}=0$.

(ii) Complex conjugation symmetry $\mathcal{C}$ : Eq. (50) remains invariant if we complex conjugate all numbers and transform $u\left(n_{x}, n_{z}, k_{y}\right) \rightarrow u^{*}\left(n_{x}, n_{z}, k_{y}\right)$. This means that the eigenstates can be chosen to be real if required.

States with $k_{y}=0$ deserve a special discussion in the case that $\vec{B}=0$. We have already seen that these states have a two-fold Kramers degeneracy. Since the Hamiltonian in Eq. (50) commutes with $\sigma^{y}$ if $k_{y}=0$, these states can be chosen to be eigenstates of $\sigma^{y}$; it then follows that the two states will have eigenvalues \pm 1 . To see this, we note that if $\psi_{1}$ satisfies $\sigma^{y} \psi_{1}=\psi_{1}$ with eigenvalue +1 , the other eigenstate will be $\psi_{2}=\sigma^{y} \psi_{1}^{*}$ and it will satisfy $\sigma^{y} \psi_{2}=-\psi_{2}$.

In the next subsection, we will present the numerical results obtained by solving Eq. (50). Since we are only using the lattice as a way to study the continuum model, 
we can trust our results only if the length scales of variation of the wave functions in all three directions are much larger than the lattice spacing $a$.

\section{NUMERICAL RESULTS}

We will begin by considering a $16 \times 16$ lattice with open boundary conditions, namely, $n_{x}$ and $n_{z}$ go from 1 to 16 . We first present the results obtained when the on-site potential $V_{n_{x}, n_{z}}=0$. The energy spectrum as a function of the dimensionless parameter $k_{y} a$ lying in the range $[-\pi / 5, \pi / 5]$ is shown in Fig. 3. [In this section, we are only going to look at states with a limited range of $k_{y} a$ close to zero, since it is only for this range that the numerical results based on our lattice model can be expected to match with those obtained using a continuum Hamiltonian]. For each value of $k_{y}$, there are 1024 energy levels. We see that there is only a small energy gap at $k_{y}=0$ and $E=0$; this small gap is due to the finite width of the system, namely, the fact that the minimum value of $k_{x}$ or $k_{z}$ is about $\pi / 16$ rather than zero. The gap opens up linearly as $\left|k_{y}\right|$ increases. Looking at the wave functions, we discover that the states closest to $E=0$ are peaked at the surfaces, namely, near $n_{x}$ or $n_{z}$ equal to 1 or 16 , while those further away from $E=0$ are bulk states. More precisely, the states lying within the range $-m<E<m$ (where $m=0.28 \mathrm{eV}$ is half the bulk gap) are surface states, while the states lying outside this range are bulk states. It is visually clear from Fig. 3 that the density of states for surface states is less than that for bulk states. This is because, for a given value of $k_{y}$, the surface states are labeled by only one other momentum ( $k_{x}$ on the top surface and $k_{z}$ on the side surface), while the bulk states are labeled by two other momenta (both $k_{x}$ and $k_{z}$ ); hence the number of bulk states is much larger than the number of surface states.

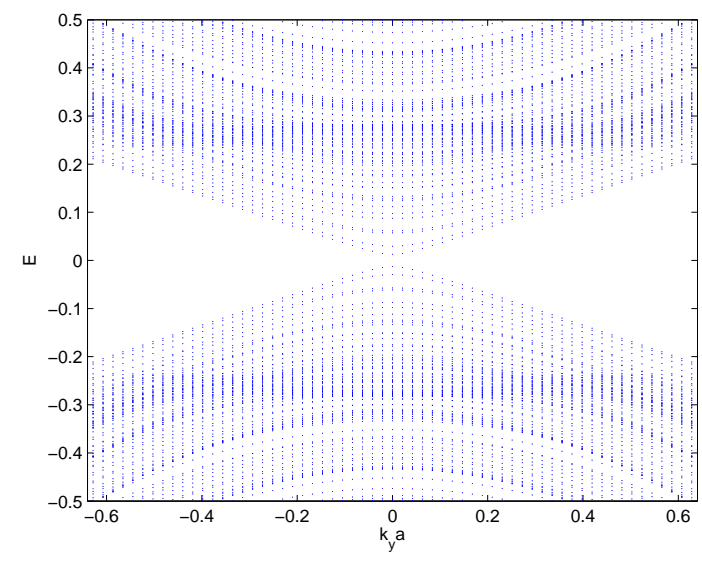

FIG. 3: (Color online) $E$ (in $\mathrm{eV}$ ) versus $k_{y} a$ lying in the range $[-\pi / 5, \pi / 5]$ for a $16 \times 16$ lattice, with $V_{n_{x}, n_{z}}=0$ at all sites.
We now introduce a potential at one corner of the lattice. We have considered three cases corresponding to $V_{n_{x}, n_{z}}=0.5,1$ and $1.5 \mathrm{eV}$ at $\left(n_{x}, n_{z}\right)=(16,16)$ and zero everywhere else. The energy spectra as functions of $k_{y} a$ are shown in Figs. 4 (a-c). In each figure we see a set of states (shown by thick blue dots) which stay close to $E=0$ for $k_{y} a$ lying in the range $[-\pi / 5, \pi / 5]$. We find that these states are non-degenerate, except at $k_{y}=0$ where there is a Kramers degeneracy. The wave functions of these states are localized at the corner of the lattice where the potential is present; hence we will refer to these as corner states in this section. We note that $n_{z}=16$ $\left(n_{x}=16\right)$ correspond to the top (side) surface discussed in Sec. II A; hence the corner $\left(n_{x}, n_{z}\right)=(16,16)$ corresponds to the edge between those two surfaces discussed in Sec. II C.
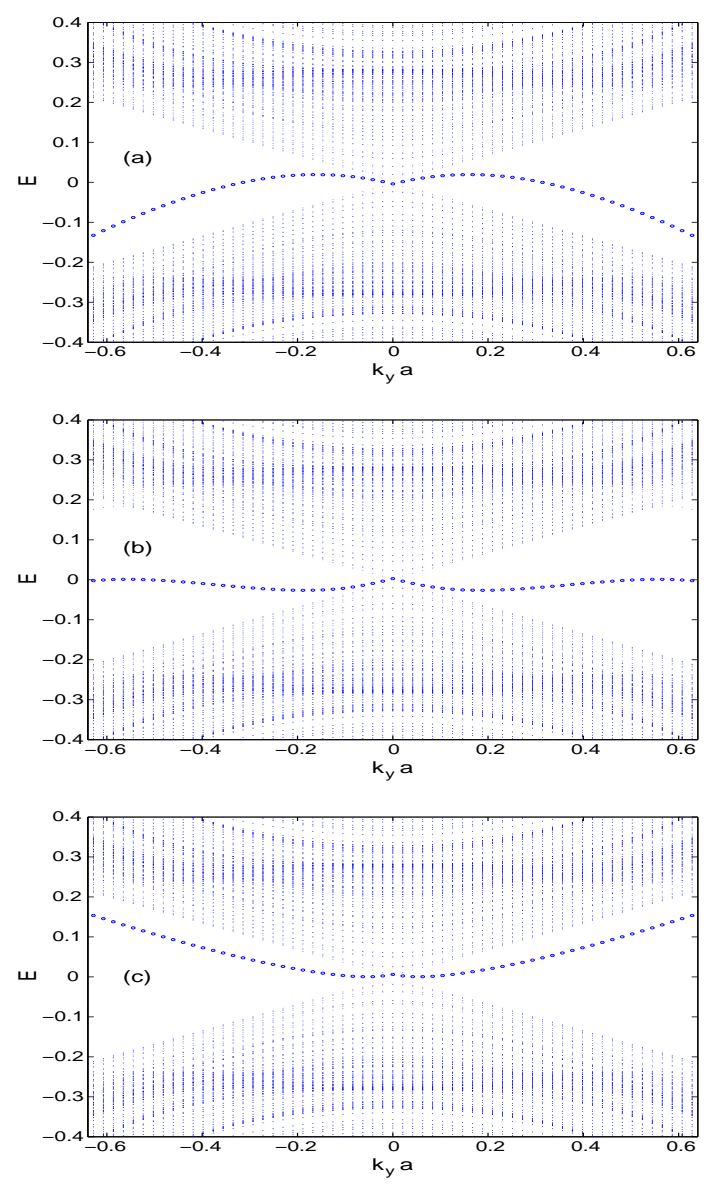

FIG. 4: (Color online) $E$ (in $e V$ ) versus $k_{y} a$ in the range $[-\pi / 5, \pi / 5]$ for a $16 \times 16$ lattice, with $V_{16,16}$ being equal to (a) 0.5 , (b) 1 and (c) $1.5 \mathrm{eV}$.

The dispersion found numerically agrees qualitatively (but not quantitatively) with the one found analytically in Sec. II $\mathrm{C}$ where it was shown that the energy is of the form $E=v\left|k_{y}\right|$, where $v$ changes from negative to positive values as the potential parameterized by $\alpha$ is increased. Namely, we see from Figs. 4 (a-c) that the 
energy is the same for $k_{y}$ and $-k_{y}$, and that it curves downwards for $V=0.5 \mathrm{eV}$, is quite flat for $V=1 \mathrm{eV}$ and curves upwards for $V=1.5 \mathrm{eV}$. Thus the numerical calculation on the lattice confirms the result obtained analytically that the parameter $\alpha$ describes a potential at the edge between two surfaces and leads to the presence of edge states whose dispersion changes with $\alpha$. We should remark here that the numerical results are not expected to quantitatively match the analytical results for very small values of $\left|k_{y} a\right|$ where the decay length of the corner states becomes of the order of the system size (namely, these cease to be corner states). Further, the fact that the numerically obtained dispersion is nonlinear rather than linear in $k_{y}$ may be due to the terms proportional to $B_{1}$ and $B_{2}$ in Eq. (49).

We note that any potential on a lattice necessarily has a finite width of at least one lattice spacing, and is therefore not identical to a $\delta$-function potential. Hence a lattice model with a potential will sometimes show additional sets of corner states which lie away from $k_{y} a=0$. This is in contrast to the continuum model with a $\delta$ function barrier where we get only one set of corner states whose momenta come arbitrarily close to $k_{y}=0$ where the energy also tends to zero. (We recall that states obtained in a lattice calculation have a counterpart in the continuum only in the limit $k_{y} a \rightarrow 0$ ). An example of such additional states is shown by the two sets of red triangles in Fig. 5. In this figure the parameters are the same as in Fig. 4 (b) but the range of $k_{y} a$ has been increased to $[-1,1]$ in order to show these extra states. We also note that the states shown by large blue dots which approach $E=0$ as $k_{y} \rightarrow 0$ go quite far away from zero energy as $k_{y} a$ becomes large; this is due to the non-linear terms proportional to $B_{1}$ and $B_{2}$ in Eq. (49). In the rest of our discussion we will only concentrate on the corner states which lie close to zero momentum and zero energy since these are the direct counterparts of the edge states found in the continuum model.

A surface plot of the probability for the wave function of the corner state with $k_{y} a=0.2$ is shown in Fig. 6. We consider each lattice site labeled as $\left(n_{x}, n_{z}\right)$ and calculate the probability density namely, $P_{n_{x}, n_{z}, k_{y}}=$ $\psi_{n_{x}, n_{z}, k_{y}}^{\dagger} \psi_{n_{x}, n_{z}, k_{y}}$. This is then shown as a surface plot versus $n_{x}$ and $n_{z}$.

It is interesting to look at the spin structure of the corner state, namely, the expectation values of $S^{a}=\sigma^{a} / 2$. Fig. 7 shows the expectation values $\left\langle S^{x}\right\rangle,\left\langle S^{y}\right\rangle$ and $\left\langle S^{z}\right\rangle$ as functions of $k_{y} a$ for the corner state of a $20 \times 20$ lattice with $V_{20,20}=0.5,1$ and 1.5 . (The rapid variations of some of the quantities near $k_{y}=0$ may not be significant since, as remarked earlier, the decay length of the corner state becomes of the order of the system size when $k_{y} \rightarrow 0$. The properties of the corner states can no longer be found reliably when that happens). The figures shows that $\left\langle S^{x}\right\rangle$ and $\left\langle S^{z}\right\rangle$ flip sign under $k_{y} \rightarrow-k_{y}$, while $\left\langle S^{y}\right\rangle=0$ for all values of $k_{y}$. (An exception to this occurs at $k_{y}=0$ where there are two states which are eigenstates of $S^{y}$ with eigenvalues $\pm 1 / 2$ ). These state-

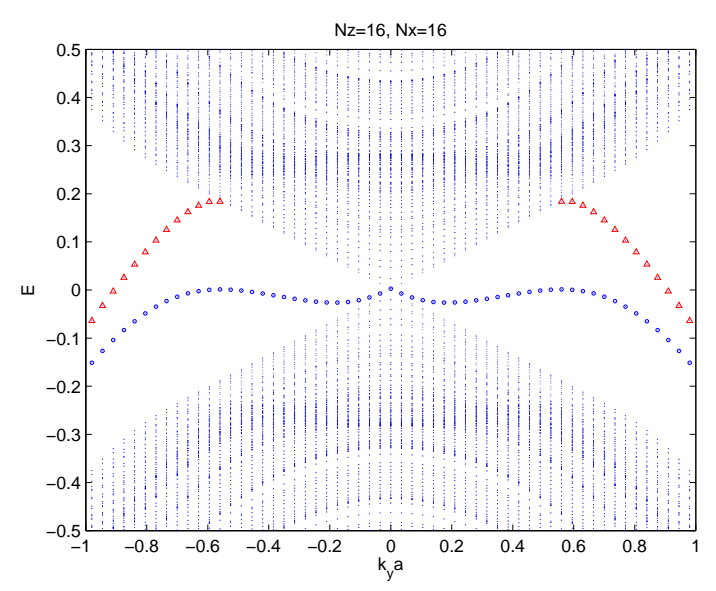

FIG. 5: (Color online) $E$ (in $e V$ ) versus $k_{y} a$ in the range $[-1,1]$ for a $16 \times 16$ lattice, with $V_{n_{x}, n_{z}}=1 \mathrm{eV}$ at $\left(n_{x}, n_{z}\right)=$ $(16,16)$. Two sets of corner states are shown by large blue dots (these states exist close to $k_{y}=0$ ) and red triangles (these only exist for $\left|k_{y} a\right| \gtrsim 0.6$ ).

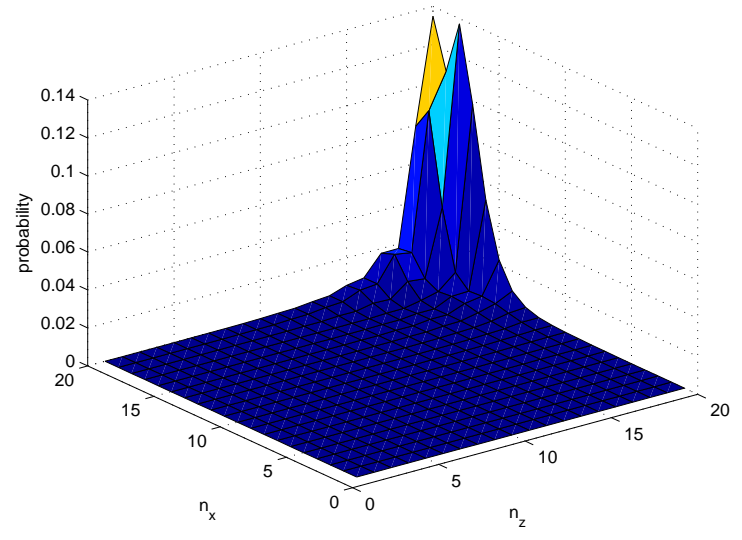

FIG. 6: (Color online) Surface plot of the probability density versus $n_{x}$ and $n_{z}$ for the corner state with $k_{y} a=0.2$, for a $20 \times 20$ lattice with $V_{20,20}=1 \mathrm{eV}$.

ments can be proved as follows. The time-reversal symmetry $u\left(n_{x}, n_{z}, k_{y}\right) \rightarrow \sigma^{y} u^{*}\left(n_{x}, n_{z},-k_{y}\right)$ imply that $\left\langle S^{x}\right\rangle$ and $\left\langle S^{z}\right\rangle$ change sign under $k_{y} \rightarrow-k_{y}$ because $\sigma^{x}$ and $\sigma^{z}$ anticommute with $\sigma^{y}$. The complex conjugation symmetry $\mathcal{C}$ discussed in Sec. III implies that $\left\langle S^{y}\right\rangle=0$ for each value of $k_{y}$ because $\sigma^{y *}=-\sigma^{y}$.

Fig. 7 implies that the nature of the spin-momentum locking of the corner states differs from that of the surface states. The direction of spin lies in the $x-z$ plane and depends on both the sign and magnitude of $k_{y}$ and the barrier potential $V$. However the property that the sign of $\langle\vec{S}\rangle$ flips under $k_{y} \rightarrow-k_{y}$ holds for both surface states and corner states.

Next, we find that a magnetic field along the $x$ di- 

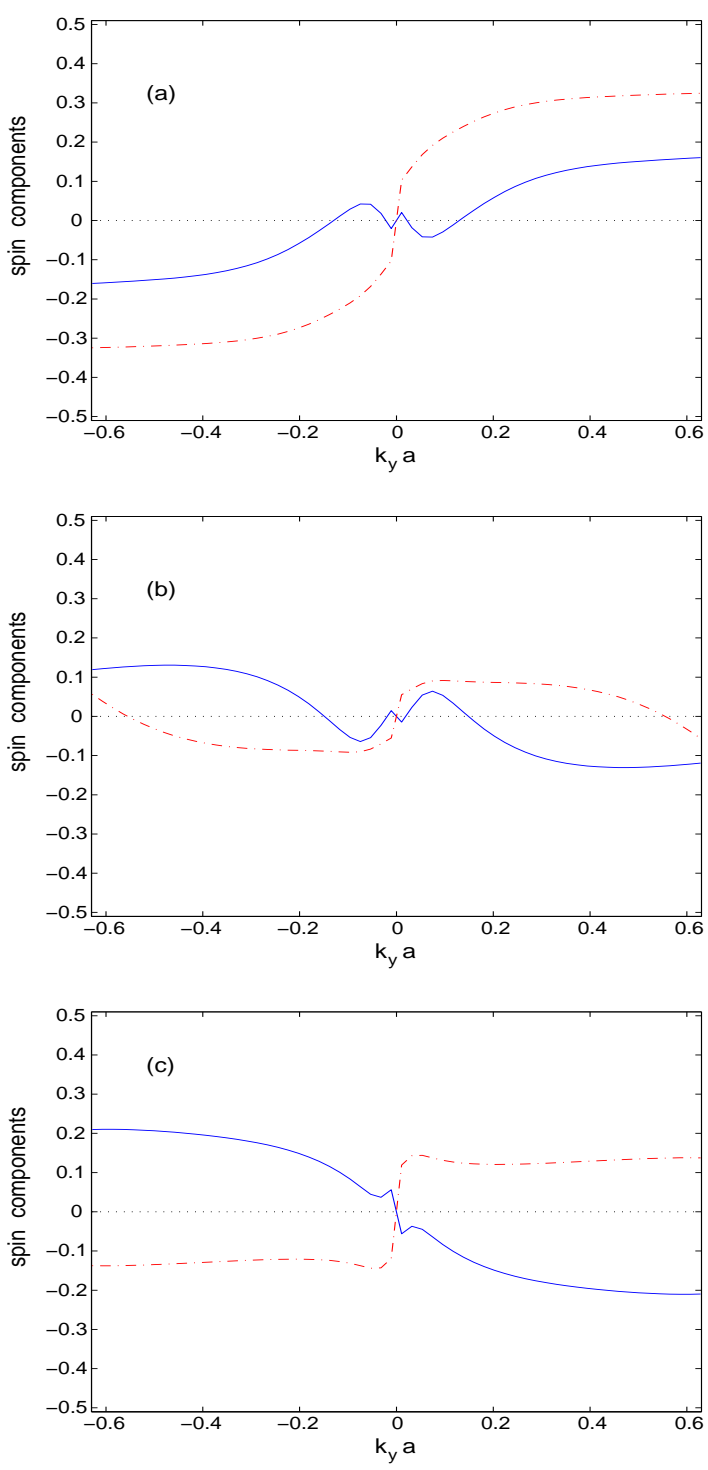

FIG. 7: (Color online) Spin components $S^{x}$ (blue solid), $S^{y}$ (black dotted) and $S^{z}$ (red dash dot) versus $k_{y} a$ for the corner states of a $20 \times 20$ lattice, with $V_{20,20}$ being equal to (a) 0.5 , (b) 1 and (c) $1.5 \mathrm{eV}$.

rection can also produce corner states with finite momentum. The energy-momentum dispersion is shown in Fig. 8. The energy of these states goes to zero in the thermodynamic limit as we will discuss below. For each value of $k_{y}$ and $B_{x}$ consistent with Eq. (46), there are two states each of which lies at two out of the four corners. [There are certain linear combinations of these two states which lie at only one of the corners. Due to the finite size of our systems, there is tunneling between the two corners; hence the energies of the two states split to give some non-zero values $\pm E$ instead of lying exactly at zero. The splitting goes to zero exponentially as the system size is increased]. Fig. 9 shows the probability density for states appearing at the corners $\left(n_{x}, n_{z}\right)=(0,20)$ and $(20,20)$ for $g \mu_{B} B_{x}=-0.2$ and $k_{y} a=0.1$. Note that the decay lengths of these states along the $x$ and $z$ directions are quite different from each other, as suggested by Eq. (47).

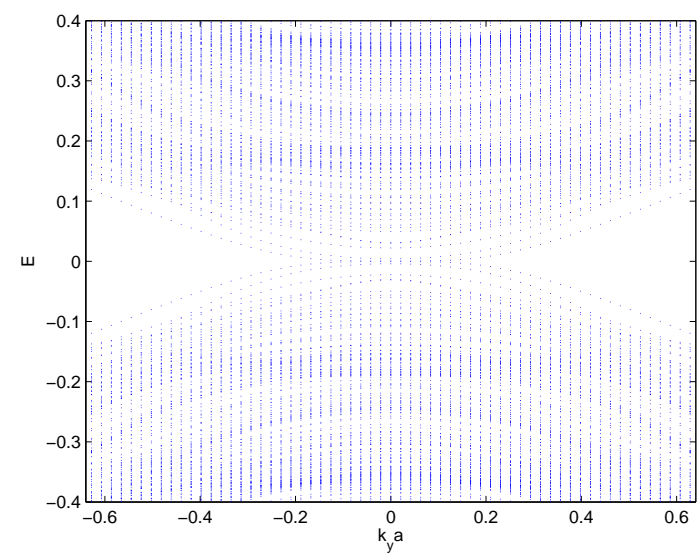

FIG. 8: (Color online) $E$ (in $e V$ ) versus $k_{y} a$ for a $16 \times 16$ lattice with $g \mu_{B} B_{x}=-0.2$. The corner states have an energy close to zero in the range $\left|k_{y} a\right| \lesssim 0.15$.

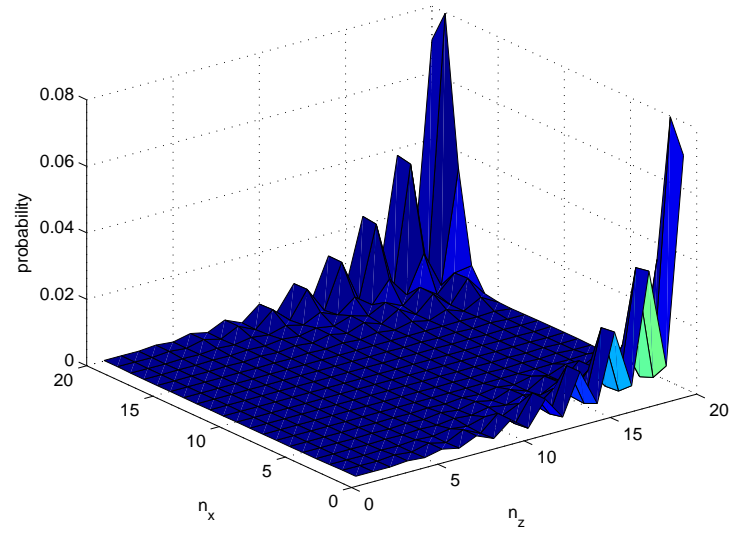

FIG. 9: (Color online) Surface plot of the probability versus $n_{x}$ and $n_{z}$ for states at the corners $(0,20)$ and $(20,20)$ with $k_{y} a=0.1$, for a $20 \times 20$ lattice with $g \mu_{B} B_{x}=-0.2$.

Fig. 10 shows the expectation values $\left\langle S^{x}\right\rangle,\left\langle S^{y}\right\rangle$ and $\left\langle S^{z}\right\rangle$ as functions of $k_{y} a$ for the states localized at the corner $\left(n_{x}, n_{z}\right)=(20,20)$ of a $20 \times 20$ lattice with $b_{x} \equiv g \mu_{B} B_{x}=-0.2$. We have numerically constructed such states by taking linear combinations of two energy eigenstates each of which has states at two corners as shown in Fig. 9. We have chosen $k_{y}>0$ and $b_{x}<0$ so that the states appears near the corner at $(20,20)$ where the analysis around Eqs. (43-48) is applicable. In accordance with the statement made after Eq. (48) for $k_{y}>0$ and $b_{x}<-2 v_{p}\left|k_{y}\right|$, we see that $\left\langle S^{x}\right\rangle=\left\langle S^{y}\right\rangle=0$ while 
$\left\langle S^{z}\right\rangle<0$. Interestingly we find that $\left\langle S^{z}\right\rangle$ varies almost linearly with $k_{y}$. If we choose $k_{y}<0$ and $b_{x}<0$ with $b_{x}<-2 v_{p}\left|k_{y}\right|$, we find states appearing at the other two corners $(0,0)$ and $(20,0)$. To summarize, we see that a magnetic field in the $x$ direction can produce corner states with a momentum in the $y$ direction and a spin in the $z$ direction.

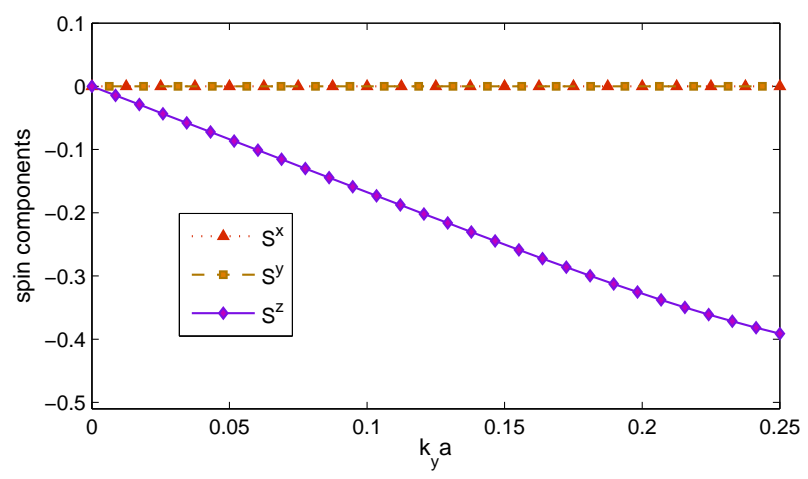

FIG. 10: (Color online) Spin components $S^{x}, S^{y}$ and $S^{z}$ versus $k_{y} a$ in the range $[0,0.25]$ for the states at the corner $(20,20)$ of a $20 \times 20$ lattice with $g \mu_{B} B_{x}=-0.2$. The lines for $\left\langle S^{x}\right\rangle=\left\langle S^{y}\right\rangle=0$ coincide.

\section{SUMMARY AND DISCUSSION}

In this paper, we have studied various properties of the surface and edge states of a three-dimensional TI, both analytically using a continuum theory and numerically using a lattice model. Analytically, we first found the Hamiltonians which govern the states on different surfaces (in particular, on the top surface and a side surface) of the system starting from a continuum bulk Hamiltonian which involves four bands. We then found the most general boundary condition that the wave function has to satisfy at the junction of the top and a side surface; this turns out to involve a single parameter $\alpha$ which can be interpreted as arising from a barrier running along the junction. Using the surface Hamiltonians and the boundary condition, we obtained the following results.

(i) We found the dependence of the differential conductance $G$ through the junction on the barrier parameter $\alpha$. For $\alpha=0$, the Hamiltonians on the top and side surfaces are related by the same unitary transformation as the one relating the wave functions. Using this, we have shown that there is no reflection from the junction and the transmission is perfect; hence $G$ has the maximum possible value at $\alpha=0$. The minimum value of $G$ occurs at $\alpha= \pm \pi / 2$.

(ii) We have shown that the barrier gives rise to edge states which propagate as plane waves along the junction and decay exponentially away from the junction.
The energy, decay length and spin of these states are related to the momentum $k_{y}$ along the junction. In particular, the energy is given by $v\left|k_{y}\right|$, where $v$ depends on the barrier parameter $\alpha$. For the special values of the barrier parameter $\alpha= \pm \pi / 2$, the energy is zero for all $k_{y}$. For any value of $\alpha$, the energy of the edge states is less than that of the surface states with the same momentum $k_{y}$. This implies that momentum conserving scattering cannot cause a transition between an edge state and a surface state. However momentum non-conserving scattering (which may arise from impurities lying on or near the barrier) can cause such transitions.

(iii) We have shown that a magnetic field applied in a direction perpendicular to the junction of the top and a side surface can also give rise to localized edge states if a dimensionless ratio between the field $B_{x}$ and the edge momentum $k_{y}$ is larger than some value. These edge states have zero energy and a spin which points in the $\pm z$ direction, i.e., perpendicular to both the magnetic field and the momentum along the junction.

To understand better the properties of the edge states found analytically from the continuum theory, we returned to the bulk Hamiltonian and studied it numerically as follows. We introduced a lattice in the $x-z$ plane keeping the $y$ direction to be a continuum with translational invariance; this enables us to maintain $k_{y}$ as a good quantum number. We used this model to numerically study different kinds of edge states. The advantages of a lattice model are that we work directly with the bulk Hamiltonian which is valid everywhere (unlike the surface Hamiltonians which are only valid when we are on a particular surface and are far from a junction of two surfaces), and we do not have to impose any unusual boundary conditions at a surface or a junction of two surfaces. Our numerical calculations gave the following results.

(iv) In the absence of any on-site potential, the energy spectrum shows two kinds of states. The states lying within the bulk gap, i.e., in the range $-m<E<m$ where $m=0.28 \mathrm{eV}$, are peaked at the surfaces and hence are surface states. The states lying outside this range are bulk states. The spectrum shows a small gap at $k_{y}=0$ due to the finite size of the system.

(v) In the presence of a potential at one corner of the $x-z$ lattice (i.e., at one edge of the three-dimensional system), a new set of states appears whose wave functions are localized at that corner. We refer to these as corner states for convenience; they correspond precisely to the edge states found using the continuum surface Hamiltonians as described above. The dispersion for these states obtained numerically for the bulk lattice system agrees qualitatively with the dispersion derived using the continuum theory for the surface states. The expectation value of the spin of these states is found to lie in the $x-z$ plane; the $y$ component of the spin is zero which can also be proved by a symmetry argument. Thus the spin is perpendicular to the momentum, although the direction of the spin of these states is different from that 
of the surface states on the adjacent surfaces.

(vi) We studied the effect of a uniform magnetic field applied along the $x$ direction, keeping only the Zeeman coupling in the lattice Hamiltonian. We find corner states under similar conditions on the relative strengths of the magnetic field and the momentum as found in the continuum model. For the choice $k_{y}>0$ and $g \mu_{B} B_{x}<0$, we found numerically that the expectation value of spin has a non-zero component only in the $-z$ direction, and its magnitude varies linearly with $k_{y}$.

We have numerically checked that edge (corner) states also occur in two other three-dimensional TIs, $\mathrm{Bi}_{2} \mathrm{Te}_{3}$ and $\mathrm{Sb}_{2} \mathrm{Te}_{3}$. For this purpose, we have used the values of the parameters in Eq. (49) given in Ref. 11 for these two materials. There are some differences between the energy spectra in the three materials. While the surface and bulk states are separated from each other in energy for $\mathrm{Bi}_{2} \mathrm{Se}_{3}$ and $\mathrm{Sb}_{2} \mathrm{Te}_{3}$, they occur at the same energies for $\mathrm{Bi}_{2} \mathrm{Te}_{3}$. (This is in agreement with known results; see Figs. 20 (b-d) of Ref. 11). Similarly, the values of the barrier potential where the edge state dispersion is almost flat and the ranges of the magnetic field value for which edge states appear are different in the three materials. However, the qualitative observation that barrier potentials at junctions of two surfaces or a magnetic field applied in the $x$ direction can give rise to edge states holds in all these materials.

Our results can be experimentally tested as follows. First of all, a barrier potential can be applied near a junction of two surfaces by placing a gate close to the junction and tuning the gate voltage. Then spin-resolved ARPES can be used to find the energy dispersion and spins of the different edge states. This method cannot be easily used in the presence of a magnetic field since the field would affect the trajectories of the electrons emitted from the surface. A second method would be to measure the local density of states using the tunneling conductance from a spin-polarized STM tip placed very close to the junction. If the local density of states is found to be higher when a potential is applied to the junction (or if a magnetic field is applied) compared to the case of no potential and no magnetic field, this would provide evidence for the edge states. Finally, we can measure the differential conductance between two point contacts placed at the two end points of the junction (i.e., the conductance in the $y$ direction); a significant value of this conductance and its variation with the barrier potential or a magnetic field would provide indirect evidence for edge states. Note that since the edge states carry a spin which is opposite for opposite edge momenta $+k_{y}$ and $-k_{y}$, a non-zero charge conductance along the junction also implies a nonzero spin conductance.

In our numerical calculations we have considered a lattice in the $x-z$ plane and continuum along the $y$ direction. It would be interesting to study the effects of impurities present at the junction on the edge states. Since the presence of impurities breaks the translational invariance along the $y$ direction, $k_{y}$ would no longer be a good quantum number. Hence it would be necessary to use a lattice model in all three directions. This may be numerically quite challenging.

It would also be interesting to use the lattice model to numerically study the effects of the orbital coupling to a magnetic field. On a lattice, such a coupling can be introduced through the phase in the couplings between nearest neighbors following the Peierls prescription. This can be used to study Aharonov-Bohm oscillations due to states which go around all the four surfaces in Fig. 1, as was studied experimentally in Ref. 51 .

\section{Acknowledgments}

We thank Sourin Das, Taylor Hughes, Nitin Samarth and Vijay Shenoy for discussions. D.S. thanks DST, India for support under Grant No. SR/S2/JCB-44/2010.
1 B. A. Bernevig, T. L. Hughes, and S.-C. Zhang, Science 314, 1757 (2006); B. A. Bernevig and S.-C. Zhang, Phys. Rev. Lett. 96, 106802 (2006).

2 L. Fu, C. L. Kane, and E. J. Mele, Phys. Rev. Lett. 98, 106803 (2007); R. Roy, Phys. Rev. B 79, 195322 (2009); J. E. Moore and L. Balents, Phys. Rev. B 75, 121306 (2007).

3 C. L. Kane and E. J. Mele, Phys. Rev. Lett. 95, 226801 (2005); ibid, Phys. Rev. Lett. 95, 146802 (2005).

4 J. C. Y. Teo, L. Fu, and C. L. Kane, Phys. Rev. B 78, 045426 (2008).

5 X. L. Qi, T. L. Hughes, and S. C. Zhang, Phys. Rev. B 78, 195424 (2008); H. Zhang, C.-X. Liu, X.-L. Qi, X. Dai, Z. Fang, and S.-C. Zhang, Nature Phys. 5, 438 (2009); C.X. Liu, X.-L. Qi, H. J. Zhang, X. Dai, Z. Fang, and S.-C. Zhang, Phys. Rev. B 82, 045122 (2010).

${ }^{6}$ Y. L. Chen, J. G. Analytis, J.-H. Chu, Z. K. Liu, S.-K. Mo, X. L. Qi, H. J. Zhang, D. H. Lu, X. Dai, Z. Fang, S. C.
Zhang, I. R. Fisher, Z. Hussain, and Z.-X. Shen, Science 325, 178 (2009); T. Zhang, P. Cheng, X. Chen, J.-F. Jia, X. Ma, K. He, L. Wang, H. Zhang, X. Dai, Z. Fang, X. Xie, and Q.-K. Xue, Phys. Rev. Lett. 103, 266803 (2009).

7 M. König, S. Wiedmann, C. Brüne, A. Roth, H. Buhmann, L. W. Molenkamp, X.-L. Qi, and S.-C. Zhang, Science 318, 766 (2007); M. König, H. Buhmann, L. W. Molenkamp, T. L. Hughes, C.-X. Liu, X.-L. Qi, and S.-C. Zhang, J. Phys. Soc. Jpn. 77, 031007 (2008); D. Hsieh, D. Qian, L. Wray, Y. Xia, Y. S. Hor, R. J. Cava, and M. Z. Hasan, Nature (London) 452, 970 (2008).

8 Y. Xia, D. Qian, D. Hsieh, L. Wray, A. Pal, H. Lin, A. Bansil, D. Grauer, Y. S. Hor, R. J. Cava, and M. Z. Hasan, Nat. Phys. 5, 398 (2009); ibid, arXiv:0907.3089 (unpublished).

9 D. Hsieh, Y. Xia, D. Qian, L. Wray, J. H. Dil, F. Meier, L. Patthey, J. Osterwalder, A. V. Fedorov, H. Lin, A. Bansil, D. Grauer, Y. S. Hor, R. J. Cava, and M. Z. Hasan, Nature 
(London) 460, 1101 (2009); P. Roushan, J. Seo, C. V. Parker, Y. S. Hor, D. Hsieh, D. Qian, A. Richardella, M. Z. Hasan, R. J. Cava, and A. Yazdani, Nature 460, 1106 (2009); D. Hsieh, Y. Xia, L. Wray, D. Qian, A. Pal, J. H. Dil, J. Osterwalder, F. Meier, G. Bihlmayer, C. L. Kane, Y. S. Hor, R. J. Cava, and M. Z. Hasan, Science 323, 919 (2009).

10 M. Z. Hasan and C. L. Kane, Rev. Mod. Phys. 82, 3045 (2010).

11 X.-L. Qi and S.-C. Zhang, Rev. Mod. Phys. 83, 1057 (2011)

12 S. Mondal, D. Sen, K. Sengupta, and R. Shankar, Phys. Rev. Lett. 104, 046403 (2010); ibid, Phys. Rev. B 82, 045120 (2010).

13 A. A. Zyuzin, M. D. Hook, and A. A. Burkov, Phys. Rev. B 83, 245428 (2011).

14 L. Fu, Phys. Rev. Lett. 103, 266801 (2009).

15 L. Fu and C. L. Kane, Phys. Rev. Lett. 100, 096407 (2008).

16 A. R. Akhmerov, J. Nilsson, and C. W. J. Beenakker, Phys. Rev. Lett. 102, 216404 (2009).

17 Y. Tanaka, T. Yokoyama, and N. Nagaosa, Phys. Rev. Lett. 103, 107002 (2009); J. Linder, Y. Tanaka, T. Yokoyama, A. Sudbo, and N. Nagaosa, Phys. Rev. Lett. 104, 067001 (2010).

18 T. Yokoyama, Y. Tanaka, and N. Nagaosa, Phys. Rev. Lett. 102, 166801 (2009).

19 A. A. Burkov and D. G. Hawthorn, Phys. Rev. Lett. 105, 066802 (2010); O. V. Yazyev, J. E. Moore, and S. G. Louie, Phys. Rev. Lett. 105, 266806 (2010); K. Nomura and N. Nagaosa, Phys. Rev. B 82, 161401 (2010); T. Yokoyama, J. Zang, and N. Nagaosa, Phys. Rev. B 81, 241410(R) (2010)

${ }^{20}$ I. Garate and M. Franz, Phys. Rev. Lett. 104, 146802 (2010); ibid, Phys. Rev. B 81, 172408 (2010).

21 K. Saha, S. Das, K. Sengupta, and D. Sen, Phys. Rev. B 84, 165439 (2011).

22 M. Guigou and J. Cayssol, Phys. Rev. B 82, 115312 (2010).

23 R. W. Reinthaler, P. Recher, and E. M. Hankiewicz, Phys. Rev. Lett. 110, 226802 (2013).

${ }^{24}$ R. Takahashi and S. Murakami, Phys. Rev. Lett. 107, 166805 (2011).

25 D. Sen and O. Deb, Phys. Rev. B, 85, 245402 (2012); Erratum, Phys. Rev. B 86 (2012) 039902(E).

26 C. Wickles and W. Belzig, Phys. Rev. B 86, 035151 (2012).

27 R. R. Biswas and A. V. Balatsky, Phys. Rev. B 83, 075439 (2011).

28 M. Alos-Palop, R. P. Tiwari, and M. Blaauboer, Phys. Rev. B 87, 035432 (2013).

${ }^{29}$ M. Sitte, A. Rosch, E. Altman, and L. Fritz, Phys. Rev. Lett. 108, 126807 (2012).

30 V. M. Apalkov and T. Chakraborty, EPL 100, 17002 (2012), and EPL 100, 67008 (2012).

31 T. Habe and Y. Asano, Phys. Rev. B 88, 155442 (2013), and Phys. Rev. B 89, 115203 (2014).

${ }^{32}$ F. Zhang, C. L. Kane, and E. J. Mele, Phys. Rev. B 86, 081303(R) (2012), and Phys. Rev. Lett. 110, 046404 (2013).

33 A. Rüegg, S. Coh, and J. E. Moore, Phys. Rev. B 88, 155127 (2013).
34 S. Modak, K. Sengupta, and D. Sen, Phys. Rev. B. 86 , 205114 (2012).

35 A. Soori, O. Deb, K. Sengupta, and D. Sen, Phys. Rev. B 87, 245435 (2013).

36 J. Nussbaum, T. L. Schmidt, C. Bruder, and R. P. Tiwari, arXiv:1405.4695.

37 B. Zhou, H.-Z. Lu, R.-L. Chu, S.-Q. Shen, and Q. Niu, Phys. Rev. Lett. 101, 246807 (2008); H.-Z. Lu, W.-Y. Shan, W. Yao, Q. Niu, and S.-Q. Shen, Phys. Rev. B 81, 115407 (2010).

38 J. Linder, T. Yokoyama, and A. Sudbo, Phys. Rev. B 80, 205401 (2009).

39 R. Egger, A. Zazunov, and A. Levy Yeyati, Phys. Rev. Lett. 105, 136403 (2010).

40 A. Kundu, A. Zazunov, A. Levy Yeyati, T. Martin, and R. Egger, Phys. Rev. B 83, 125429 (2011).

41 A. Medhi and V. B. Shenoy, J. Phys. Condens. Matter 24, 355001 (2012).

42 A. Pertsova and C. M. Canali, arXiv:1311.0691.

43 M. Neupane, A. Richardella, J. Sanchez-Barriga, S.-Y. Xu, N. Alidoust, I. Belopolski, C. Liu, G. Bian, D. M. Zhang, D. Marchenko, A. Varykhalov, O. Rader, M. Leandersson, T. Balasubramanian, T.-R. Chang, H.-T. Jeng, S. Basak, H. Lin, A. Bansil, N. Samarth, and M. Z. Hasan, arXiv:1307.5485.

44 P. G. Silvestrov, P. W. Brouwer, and E. G. Mishchenko, Phys. Rev. B 86, 075302 (2012).

45 Y. Takane and K.-I. Imura, J. Phys. Soc. Jpn. 81, 093705 (2012).

46 L. Barreto, W. Simoes e Silva, M. Stensgaard, S. Ulstrup, X.-G. Zhu, M. Bianchi, M. Dendzik, and P. Hofmann, arXiv:1302.0396.

47 U. Khanna, S. Pradhan, and S. Rao, Phys. Rev. B 87, 245411 (2013).

48 L. Brey and H. A. Fertig, Phys. Rev. B 89, 085305 (2014).

49 T. Paananen and T. Dahm, Phys. Rev. B 87, 195447 (2013).

50 T. Paananen, H. Gerber, M. Götte, and T. Dahm, New J. Phys. 16, 033019 (2014).

${ }^{51}$ H. Peng, K. Lai, D. Kong, S. Meister, Y. Chen, X.-L. Qi, S.-C. Zhang, Z.-X. Shen, and Y. Cui, Nature Materials 9, 225 (2010).

52 R. Jackiw and C. Rebbi, Phys. Rev. D 13, 3398 (1976).

53 K. Nomura and N. Nagaosa, Phys. Rev. Lett. 106, 166802 (2011); C. Niu, Y. Dai, M. Guo, W. Wei, Y. Ma, and B. Huang, Appl. Phys. Lett. 98, 252502 (2011); Y. H. Choi, N. H. Jo, K. J. Lee, J. B. Yoon, C. Y. You, and M. H. Jung, J. Appl. Phys. 109, 07E312 (2011); P. Haazen, J. Laloë, T. Nummy, H. Swagten, P. Jarillo-Herrero, D. Heiman, and J. Moodera, Appl. Phys. Lett. 100, 082404 (2012).

54 T. Yokoyama, Y. Tanaka, and N. Nagaosa, Phys. Rev. B 81, 121401(R) (2010); H. Ji, J. M. Allred, N. Ni, J. Tao, M. Neupane, A. Wray, S. Xu, M. Z. Hasan, and R. J. Cava, Phys. Rev. B 85, 165313 (2012); Q. Meng, S. Vishveshwara and T. L. Hughes, Phys. Rev. Lett. 109, 176803 (2012).

55 K.-I. Imura, A. Yamakage, S. Mao, A. Hotta, and Y. Kuramoto, Phys. Rev. B 82, 085118 (2010). 